\title{
Chronic Achilles Tendinopathy in Runners: Relationship between Pain and Tendon Vascularity and Efficacy and Safety of the Radial Extracorporeal Shock Wave Therapy
}

\author{
Antonio Ammendolia \\ Department of Medical and Surgical Sciences, University of Catanzaro “Magna Græcia”, Catanzaro, Italy \\ Email: ammendolia@unicz.it
}

Received 18 May 2015; accepted 12 June 2015; published 15 June 2015

Copyright (C) 2015 by author and Scientific Research Publishing Inc. This work is licensed under the Creative Commons Attribution International License (CC BY). http://creativecommons.org/licenses/by/4.0/

(c) (i) Open Access

\section{Abstract}

Few studies report the possible correlation pain-paratendon microvascularity during the painful phase of the chronic Achilles tendinopathy and the efficacy of the radial shock wave therapy respect to other therapies. The aim of the present longitudinal, controlled study is to demonstrate the variation of the tendon micro vascularization in athletes affected by Achilles tendinopathy and the efficacy and safety of the radial extracorporeal shock wave therapy. Twelve elite runners with Achilles tendinopathy were compared with 12 healthy amateurs, both treated by radial extracorporeal shock wave therapy in 3 sessions (1/every 3 days). VAS scale was used for pain evaluation at one and six months after treatment and a Color and Power Doppler echography was performed to observe the paratendon microvascularity before the beginning of the treatment and at one and six months after. One month after the beginning of the treatment, it was observed a decrease of the hypervascularity in all 12 subjects with tendinopathy and no variation in the control group participants. Clinically, $80 \%$ of patients referred pain relief and they were able to return to sports activity. The decrease of the paratendon microvascularity confirms the correlation between the disappearance of the pain and the normalization of the vascularity in the athletes. Moreover, radial extracorporeal shock wave therapy consented a quickly pain relief and returned to the sport. These results confirmed the efficacy and safety of this physical therapy that it could be considered a good therapeutic choice in the treatment of the chronic Achilles tendinopathy.

\section{Keywords}

Tendinopathy, Radial Shock Wave, Microvascularity 


\section{Introduction}

Achilles tendinopathy is a common cause of posterior heel pain and it's often difficult to treat. This condition is more frequent in athletes, particularly runners and jumpers, but it can affect non-athletes as well. The beginning and pathogenesis of the tendinopathy are often unknown, but intrinsic and extrinsic factors have been involved [1] [2]. Intrinsic factors include abnormal range of motion of the subtalar joints such as in hyperpronation syndrome or for a leg length discrepancy. Extrinsic factors for athletes include training errors with subsequent excessive mechanical overload. Other possible extrinsic causes are advanced age, fatigue, and obesity [3]-[8]. The literature reported the association between increased paratendon microvascularity and the symptomatic Achilles tendinopathy. Some studies based on the use of the Color Power Doppler sonography have demonstrated an increased vascular density in the Achilles paratenon that is clinically associated with chronic and insertional Achilles tendinopathy [9]-[13].

Traditional non-operative treatment of chronic insertional Achilles tendinopathy consists of rest and NSAIDS administration. Some studies suggest different therapies, such as: steroid injection, sclerosing therapy, aprotinin injection, heel lifts and custom orthoses [14]-[19]. The extracorporeal shock wave therapy has been reported to be effective for the treatment of tendinopathies [20]-[23], but until now few studies investigate the efficacy in Achilles tendinopathy.

The purpose of this study is to evaluate the correlation between increased paratendon microvascularity and pain and to determine the efficacy of radial extracorporeal shock wave therapy for the treatment of the Achilles tendinopathy in long distance runners, comparing with a control group.

\section{Methods}

After the University IRB approval, I included in the study 24 subjects, that signed an informed consent to participate own free will: 12 athletes runners (group A), 9 men and 3 women (mean age $25.9 \pm 1.2$ years) and 12 sedentary subjects (group B), 9 men and 3 women (mean age $25.6 \pm 2.3$ years). All 12 patients affected by Achilles tendinopathy in painful phase and performed medical and physical therapy for a minimum of 3 months without significant clinical improvement. The control group subjects didn't show any clinical sign of Achilles pathology. All subjects were undergone to an accurate clinical examination, to exclude subjects affected by associated pathologies, that could be a contraindication to receive the radial extracorporeal shock wave therapy, such as: coagulation diseases, local infection, or tumors. The intensity of local pain was evaluated using Visual Analogue Scale (VAS) at rest and during walking and all subjects involved underwent to an echographic exam with Color Doppler, provided by a single operator with 7.5 Mhz probe, before the beginning of the treatment (T0) and at one (T1) and six months (T2) after the end of the treatment with radial shock wave therapy. For pain evaluation the level of statistical significance was determined with student t-test.

All 24 subjects underwent to a standard protocol of radial shock wave therapy by the same operator: three sessions (1 every 3 days), during which subjects received 2000 shocks each session for a total of 6000 shocks, utilizing a mean flux of energy density of $0.22 \mathrm{~mJ} / \mathrm{mm}^{2}$ at $10 \mathrm{~Hz}$ frequency. No local anesthetic used and no subject required pain medication.

To group A subjects were asked to stop the sport activity (running), but allowed to walk normally during the treatment phase. A return to normal activities was allowed one month after the last session of radial shock wave therapy.

\section{Results}

For all subjects of the group A, the echography at T0 demonstrated the presence of an area of hypoechogenicity in the mid portion of the Achilles tendon with an increase of the microvascularity (Figure 1). In group B subjects an absence of alteration in the echographic characteristics of the tendon was demonstrated (Figure 2).

At $\mathrm{T} 1 \mathrm{a}$ significant reduction of the pain at rest, during walking and running in $58.3 \%$ of group A subjects (7 out of 12) was evident, this percentage increased to 83.3\% (10 out of 12$)$ at T2 ( $<0.0001)$ (Figure 3 ).

Color Power Doppler evaluation demonstrated a normalization of the microvascularity present before the beginning of the treatment in group A subjects (Figure 4).

No significant differences in the microvascularity of the group B subjects and no symptoms were observed (Figure 5). There were no significant side effects in either treatment groups, except for a temporary paratendon 


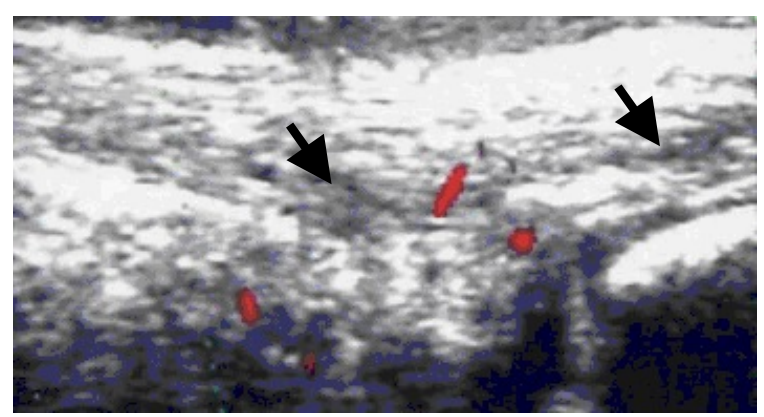

Figure 1. Group A, before treatment: diffuse hypoechogenicity areas (arrows) and thickening of the tendon with intense paratendon microvascularity.

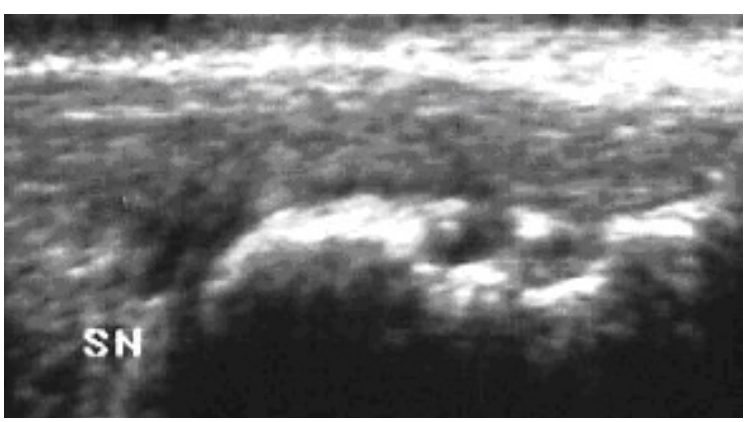

Figure 2. Group B, before treatment: normal tendon and vascularity.

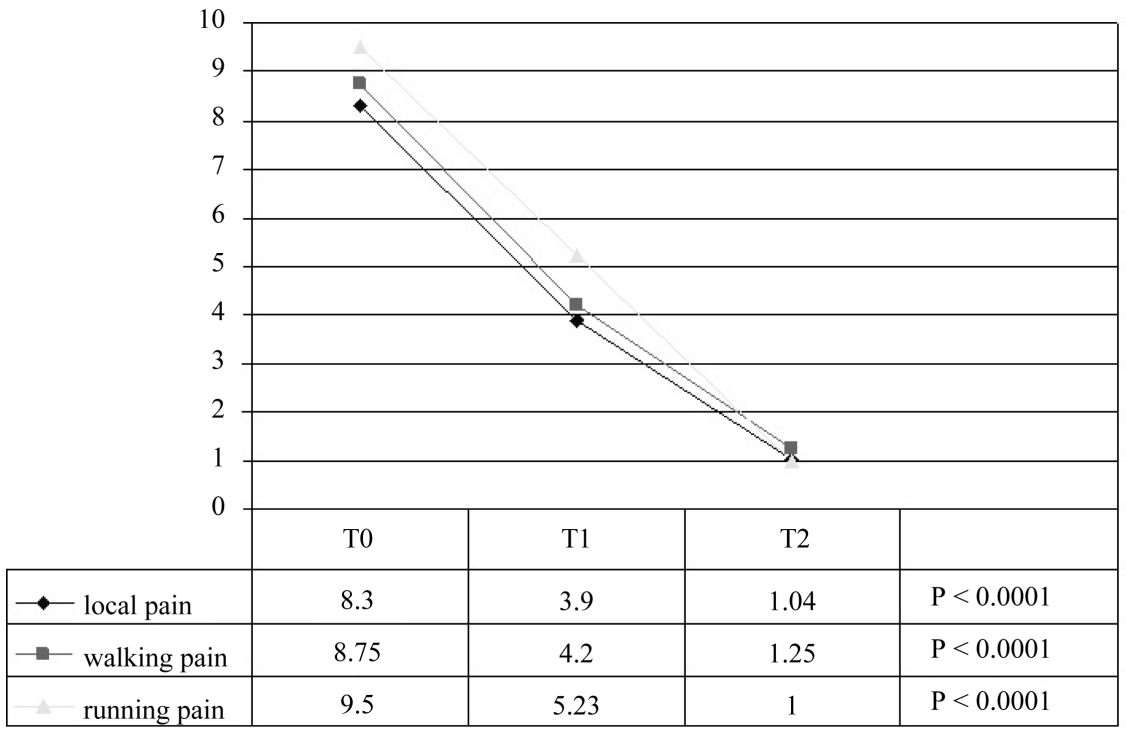

Figure 3. Group A clinical results.

edema in three subjects of the group A, which resolved by local cryotherapy in 48 hours without further consequences.

\section{Discussion}

A lot of studies evaluated the association of an increase of the microvascularity of the Achilles and patellar tendons and the intensity of the pain in patients with Achilles and patellar tendinopathy, using a Color Power 


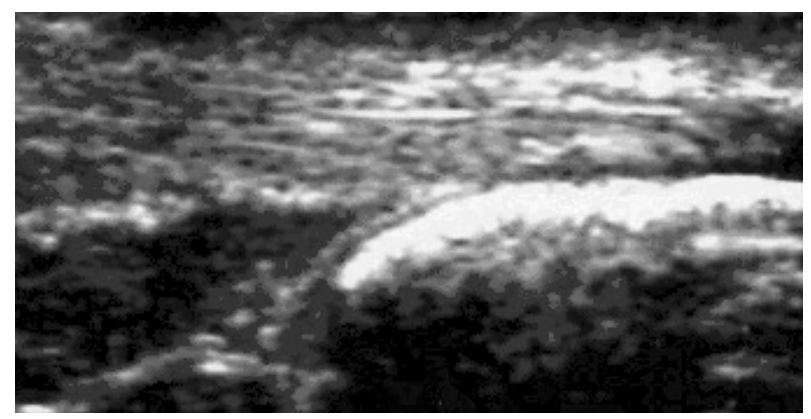

Figure 4. Group A, after 6 months: normal microvascularity and disappearance of the hypoechogenicity areas.

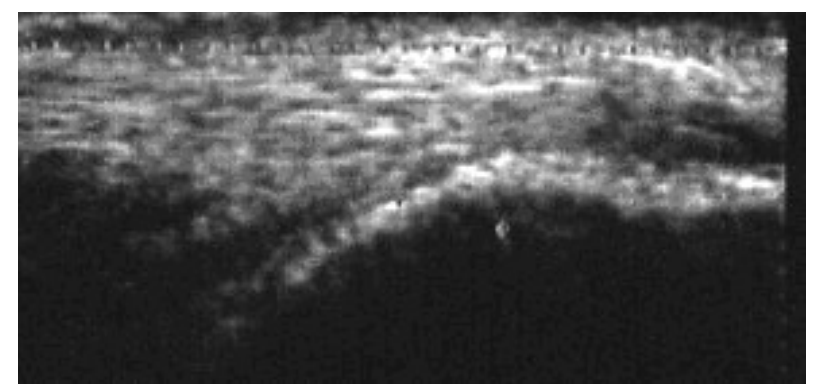

Figure 5. Group B, after 6 months: no change than before.

Doppler device. This diagnostic exam has been shown to be highly specific for the evaluation of altered microvascularity with tendinopathy [10]. Ohberg [9], that used Colored Power Doppler to demonstrate an increase in microvascularity in patients with Achilles tendinopathy, noted a reduction of pain in 8 of 12 subjects in whom he injected a sclerosing agent in the paratendon, close to the Achilles tendon insertion. In 2003 Silvestri [24] observed a hypervascularity in patients with acute tenosynovitis, compared with normal subjects. Other authors have demonstrated too an increase of the paratendon microvascularity in patients with Achilles tendinopathy, no present in asymptomatic subjects [12] [13]. Our study confirms these conclusions, because after the treatment with radial shock wave, it has been possible to observe the disappearance of the paratendon hypervascularity in all subjects of the group A, that referred pain relief, and it didn't note any difference of micro vascularization in the subjects of group B (asymptomatic).

Until now in literature there is no a "gold standard" for the treatment of the Achilles tendinopathy [25], because a lot of therapies demonstrated a different percentage of good results and time of recovery mostly long, hardly acceptable from the athletes, that have to return to the sport activity in reasonably short time. Besides in some cases side effects have occurred too [16] [17]. Injections of steroids may reduce pain, but are associated with rupture of the tendon [16]. Recently the injection of aprotine, a proteinase inhibitor, has been proposed, but the results have been unsatisfactory [17]. From some years, among different types of physical therapy for the treatment of the Achilles tendinopathy were proposed, including focused extracorporeal shock wave therapy, that is considered one of the available choice with satisfactory results in greater than eighty percent of patients [21]-[23]. This physical therapy has been shown to be useful in chronic tendinopathies, since it's able to stimulate a stagnant healing process and to decrease the nociception [26]. More recently, it's possible to read studies reporting similar clinical results using unfocused (radial) shock wave therapy [27]. Chang et al. [28] compared the effectiveness of focused shock wave therapy and a new alternative, radial shock wave, for managing of the chronic plantar fasciitis, demonstrating that radial shock wave therapy is an appropriate alternative because of its lower price and probably better effectiveness. Furia et al. [29] reported a percentage of patients with excellent or good results 12 months after treatment statistically greater in the radial extracorporeal shock wave therapy group compared to the control group $(\mathrm{P}<0.001)$ in the treatment of chronic patellar tendinopathy. Avancini-Dobrović et al. [30] evaluated the effectiveness of radial shock wave therapy on patients with calcific tendinitis of the shoulder and obtained good results after 6 months from the end of the treatment with pain relief and increase in the range of motion and muscular strength. 
To confirm the efficacy of the radial shock wave therapy, in the present study after only one month of the treatment with radial extracorporeal shock wave therapy, it's possible to observe a significant pain relief ( $\mathrm{P}<$ 0.0001 ) in over $50 \%$ of the group A subjects, that restarted sport activity. It's important to underline that the efficacy of this therapy isn't limited to a short period, but continues for several months after the end of the treatment, like demonstrated by the observation that at T2 over $80 \%$ of the athletes referred a significant further pain relief $(\mathrm{P}<0.0001)$ with return to sports activity without any significant decrease of the performance. Moreover, because it was not observed any symptom or echographic differences in group B subjects after treatment with radial shock wave, it can be stated that this therapy is safe and it cannot determine neither change in the normal tendon structure nor appearance of pain in healthy subjects, in spite of the mechanical stimulation.

\section{Conclusion}

Until now there is not any study in literature about the use of the radial shock wave therapy in the chronic Achilles tendinopathy, so, although there is a limit due to the lack number of the subjects involved, this study may be considered the first approach to demonstrate not only the efficacy of this therapeutic method but also its safety, testified by the absence of side effects in athletes and of modifications in the tendons of the healthy subjects during and after treatment. Anyway, the radial shock wave therapy is a good choice in the treatment of the Achilles tendinopathy, in particular in sportmen, because it consents a quickly relief of the pain and recovery with return to the sport.

\section{References}

[1] Rees, J.D., Wilson, A.M. and Wolman, R.L. (2006) Current Concepts in the Management of Tendon Disorders. Rheumatology, 45, 508-521. http://dx.doi.org/10.1093/rheumatology/kel046

[2] Maffulli, N., Wong, J. and Almekinders, L.C. (2003) Types and Epidemiology of Tendinopathy. Clinics in Sports Medicine, 22, 675-692. http://dx.doi.org/10.1016/S0278-5919(03)00004-8

[3] Baxter, D.E. and Zingas, C. (1995) The Foot in Running. Journal of the American Academy of Orthopaedic Surgeons, 3, 136-145.

[4] James, S.L., Bates, B.T. and Osternig, L.R. (1978) Injures to Runners. American Journal of Sports Medicine, 6, 40-50. http://dx.doi.org/10.1177/036354657800600202

[5] Jones, D.C. and James, S.L. (1987) Overuse Injures of the Lower Extremity: Shin Splints, Iliotibial Band Friction Syndrome and Exertional Compartment Syndromes. Clinics in Sports Medicine, 6, 273-290.

[6] Kolodziej, P., Glisson, R.R. and Nunley, J.A. (1999) Risk of Avulsion of the Achilles Tendon after Partial Excision for Treatment of Insertional Tendonitis and Haglund's Deformity. Foot Ankle International, 20, 433-437. http://dx.doi.org/10.1177/107110079902000707

[7] Myerson, M. and McGarvey, W. (1999) Disorders of the Achilles Insertion and Achilles Tendonitis. Instructional Course Lectures, 48, 211-218.

[8] Nigg, B.M. (2001) The Role of Impact Forces and Foot Pronation: A New Paradigm. Clinical Journal of Sport Medicine, 11, 2-9. http://dx.doi.org/10.1097/00042752-200101000-00002

[9] Öhberg, L., Lorentzon, R. and Alfredson, H. (2001) Neovascularization in Achilles Tendons with Painful Tendinosis but Not in Normal Tendons: An Ultrasonographic Investigation. Knee Surgery, Sports Traumatology, Arthroscopy, 9, 233-238. http://dx.doi.org/10.1007/s001670000189

[10] Reiter, M., Ulreich, N., Dirisamer, A., Tscholakoff, D. and Bucek, R.A. (2004) Colour and Power Doppler Sonography in Symptomatic Achilles Tendon Disease. International Journal of Sports Medicine, 25, 301-305. http://dx.doi.org/10.1055/s-2004-815828

[11] Alfredson, H. (2005) The Chronic Painful Achilles and Patellar Tendon: Research on Basic Biology and Treatment. Scandinavian Journal of Medicine and Science in Sports, 15, 252-259. http://dx.doi.org/10.1111/j.1600-0838.2005.00466.x

[12] Richards, P.J., Win, T. and Jones, P.W. (2005) The Distribution of Microvascular Response in Achilles Tendinopathy Assessed by Colour and Power Doppler. Skeletal Radiology, 34, 336-342. http://dx.doi.org/10.1007/s00256-004-0834-2

[13] Knobloch, K., Kraemer, R., Lichtenberg, A., Jagodzinski, M., Gossling, T., Richter, M., Zeichen, J., Hufner, T. and Krettek, C. (2006) Achilles Tendon and Paratendon Microcirculation in Midportion and Insertional Tendinopathy in Athletes. American Journal of Sports Medicine, 34, 92-97. http://dx.doi.org/10.1177/0363546505278705

[14] Alfredson, H. and Lorentzon, R. (2000) Chronic Achilles Tendinosis: Recommendations for Treatment and Prevention. 
Sports Medicine, 29, 135-146. http://dx.doi.org/10.2165/00007256-200029020-00005

[15] Öhberg, L. and Alfredson, H. (2003) Sclerosing Therapy in Chronic Achilles Tendon Insertional Pain-Results of a Pilot Study. Knee Surgery, Sports Traumatology, Arthroscopy, 11, 339-343. http://dx.doi.org/10.1007/s00167-003-0402-7

[16] Koenig, M.J., Torp-Pedersen, S., Ovistgaard, E., Terslev, L. and Bliddal, H. (2004) Preliminary Results of Colour Doppler-Guided Intratendinous Glucocorticoid Injection for Achilles Tendonitis in Five Patients. Scandinavian Journal of Medicine and Science in Sports, 14, 100-106. http://dx.doi.org/10.1046/j.1600-0838.2003.00364.x

[17] Brown, R., Orchard, J., Kinchington, M., Hooper, A. and Nalder, G. (2006) Aprotinin in the Management of Achilles Tendinopathy: A Randomised Controlled Trial. British Journal of Sports Medicine, 40, 275-279. http://dx.doi.org/10.1136/bjsm.2005.021931

[18] Saltzman, C.L. and Tearse, D.S. (1998) Achilles Tendon Injures. Journal of the American Academy of Orthopaedic Surgeons, 6, 316-325.

[19] Sorosky, B., Press, J., Plastaras, C. and Rittenburg, J. (2004) The Practical Management of Achilles Tendinopathy. Clinical Journal of Sport Medicine, 14, 40-44. http://dx.doi.org/10.1097/00042752-200401000-00007

[20] Rompe, J.D., Kirkpatrick, J.D., Kullmer, K., Schwitalle, M. and Krischek, O. (1998) Dose-Related Effects of Shock Waves on Rabbit Tendon Achilles. A Sonographic and Histological Study. The Journal of Bone and Joint Surgery, 80, 546-552. http://dx.doi.org/10.1302/0301-620X.80B3.8434

[21] Perlick, L., Schiffmann, R., Kraft, C.N., Wallny, T. and Diedrich, O. (2002) Extracorporeal Shock Wave Treatment of the Achilles Tendonitis: Experimental and Preliminary Clinical Results. Zeitschrift für Orthopädie, 140, 275-280. http://dx.doi.org/10.1055/s-2002-32475

[22] Chen, Y.J., Wang, C.J., Yang, K.D., Kuo, Y.R., Huang, H.C., Huang, Y.T., Sun, Y.C. and Wang, F.S. (2004) Extracorporeal Shock Waves Promote Healing of Collagenase-Induced Achilles Tendonitis and Increase TGF-Beta 1 and IGF-I Expression. Journal of Orthopaedic Research, 22, 854-861. http://dx.doi.org/10.1016/j.orthres.2003.10.013

[23] Furia, J.P. (2006) High-Energy Extracorporeal Shock Wave Therapy as a Treatment for Insertional Achilles Tendinopathy. American Journal of Sports Medicine, 34, 733-740. http://dx.doi.org/10.1177/0363546505281810

[24] Silvestri, E., Biggi, E., Molfetta, L., Avanzino, C., La Paglia, E. and Garlaschi, G. (2003) Power Doppler Analysis of Tendon Vascularization. International Journal of Tissue Reactions, 25, 149-158.

[25] Lopez, R.G. and Jung, H.G. (2015) Achilles Tendinosis: Treatment Options. Clinics in Orthopedic Surgery, 7, 1-7. http://dx.doi.org/10.4055/cios.2015.7.1.1

[26] Ochiai, N., Ohtori, S., Sasho, T., Nakagawa, K., Takahashi, K., Takahashi, N., Murata, R., Takhashi, K., Moriya, H., Wada, Y. and Saisu, T. (2007) Extracorporeal Shock Wave Therapy Improves Motor Dysfunction and Pain Originating from Knee Osteoarthritis in Rats. Osteoarthritis and Cartilage, 15, 1093-1096. http://dx.doi.org/10.1016/j.joca.2007.03.011

[27] Foldager, C.B., Kearney, C. and Spector, M. (2012) Clinical Application of Extra Corporeal Shock Wave Therapy in Orthopedics: Focused versus Unfocused Shockwaves. Ultrasound in Medicine \& Biology, 38, 1673-1680. http://dx.doi.org/10.1016/j.ultrasmedbio.2012.06.004

[28] Chang, K.V., Chen, S.Y., Chen, W.S., Tu, Y.K. and Chien, K.L. (2012) Comparative Effectiveness of Focused Shock Wave Therapy of Different Intensity Levels and Radial Shock Wave Therapy for Treating Plantar Fasciitis: A Systematic Review and Network Meta-Analysis. Archives of Physical Medicine and Rehabilitation, 93, 1259-1268. http://dx.doi.org/10.1016/j.apmr.2012.02.023

[29] Furia, J.P., Rompe, J.D., Cacchio, A., Del Buono, A. and Maffulli, N. (2013) A Single Application of Low-Energy Radial Extracorporeal Shock Wave Therapy Is Effective for the Management of Chronic Patellar Tendinopathy. Knee Surgery, Sports Traumatology, Arthroscopy, 21, 346-350. http://dx.doi.org/10.1007/s00167-012-2057-8

[30] Avancini-Dobrović, V., Frlan-Vrgoc, L., Stamenković, D., Pavlović, I. and Vrbanić, T.S. (2011) Radial Extracorporeal Shock Wave Therapy in the Treatment of Shoulder Calcific Tendinitis. Collegium Antropologicum, 35, 221-225. 